\title{
THE PERFORMANCE OF AN L3 HADRON CALORIMETER PROTOTYPE MODULE WITH BGO
}

\author{
C. CHEN, H.S. CHEN, X.G. LI, Y.S. LU, Y.F. MAO, X.W. TANG, K.L. TUNG, C.N. WANG, K.S. \\ YANG, Q.Y. YANG and H.L. ZHUANG \\ Institute of High Energy Physics, Academia Sinica, Beijing, P.R. China
}

H. HOFER, P. LECOMTE and P. LeCOULTRE

Institut für Hochenergiephysik, Eidgenössische Technische Hochschule, Zürich, Switzerland

\author{
Yu. GORODKOV, V. KUTSENKO, V. PLYASKIN, E. SHUMILOV and V. TCHUDAKOV \\ ITEP, Moscow, USSR
}

\author{
W. BÖHLEN, H.T. LI, B. SPIESS, J. ULBRICHT and S.Y. ZHANG \\ Paul Scherrer Institut (formerly Eidgenössisches Institut für Reaktorforschung), Würenlingen, Switzerland
}

\author{
T. AZEMOON, R.C. BALL, S. GOLDFARB, L.W. JONES, G.B. MILLS and B.P. ROE \\ University of Michigan, Ann Arbor, Michigan, USA
}

Received 9 May 1988

A prototype of a gas sampling uranium module of the $\mathrm{L} 3$ hadron calorimeter was built and subsequently tested with pion beams of energies between 4 and $20 \mathrm{GeV}$. The construction of the proportional chambers, the assembling of the module, the readout electronics, and the beam test are briefly described. The energy resolution of the calorimeter module alone was measured to be $30.5 \pm 3 \%$ at $6 \mathrm{GeV}, 21 \pm 2 \%$ at $10 \mathrm{GeV}$ and $18 \pm 2 \%$ at $20 \mathrm{GeV}$. For the module together with a BGO crystal matrix in front, the resolution was $29 \pm 3 \%$ at $4 \mathrm{GeV}, 21 \pm 2 \%$ at $10 \mathrm{GeV}$, and $17 \pm 2 \%$ at $20 \mathrm{GeV}$.

\section{Introduction}

The calorimeters for the L3 experiment to be used at the CERN Large Electron Positron collider, LEP, consist of a bismuth germanate (BGO) electromagnetic calorimeter followed by a uranium hadron calorimeter $[1,2]$. The hadron calorimeter [2,3] consists of three major parts: the central barrel, the endcaps, and the muon filter which surrounds the barrel. The barrel part and the endcaps are made of uranium absorbers and sampling wire chambers working in the proportional mode, which allows the calorimeter to be embedded in the high magnetic field of the L3 detector. It provides a measurement of hadron energy flow and an identification of muons. The barrel part is divided into nine rings along the beam axis with sixteen modules in each ring.

We have previously reported [4,5] test beam measurements of the characteristics of uranium calorimeters. A realistic prototype of the module to be used in the L3 detector was built. The module with and without a matrix of $10 \times 10 \mathrm{BGO}$ crystals in front of it was tested in pion beams with energies between 4 and 20 GeV at the CERN SPS in 1986.

In this paper, the construction of the proportional chambers, the assembling of the module, the readout electronics and the test beam setup are briefly described. The test beam results and a comparison with the Monte Carlo calculations including measurements of the linearity, the energy resolution and the spatial resolution are reported. More detailed descriptions of the chamber and module construction along with further test beam results will be presented later.

\section{Module structure and chamber construction}

The module consists of one stainless steel plate and 58 uranium plates interleaved with 60 planes of wire chambers as illustrated in fig. 1 . The uranium plates are made of depleted uranium with a thickness of $5 \mathrm{~mm}$. They are plated with $\mathrm{Cu}-\mathrm{Ni}$. The wire chambers operate in the proportional mode with analog pulse height 
readout. The absorber plates and chamber planes were contained in a gas-tight stainless steel module housing in which they were held rigidly and which contained appropriate signal, high voltage, and gas distribution systems. The module and its mounting in the final L3 detector and in this test beam included $4 \mathrm{~cm}$ of stainless steel in front of the first chamber. The whole module is also flushed with chamber gas. The total thickness of the uranium calorimeter module is 3.9 proton absorption lengths [11]. The BGO crystal matrix, which has been described elsewhere [7], has a thickness of 1.1 proton absorption lengths.

The proportional chambers consist of closed rectangular brass tubes with $5 \times 10 \mathrm{~mm}^{2}$ cross section. The gamma rays and electrons resulting from the natural radioactivity of ${ }^{238} \mathrm{U}$ produce background signals in the chambers. The sensitive volume of the chambers is shielded by $1 \mathrm{~mm}$ of brass from the uranium. The background counting rate for the chambers in the module is approximately $40 \mathrm{~Hz} / \mathrm{cm}^{2}$. The gold-plated tungsten anode wire is $50 \mu \mathrm{m}$ in diameter. The wires of neighboring chamber planes are perpendicular. The gas mixture used consists of $80 \%$ argon and $20 \% \mathrm{CO}_{2}$ at atmospheric pressure.

The number of readout channels is reduced by grouping the chamber wires into towers. Aided by Monte Carlo simulation of hadron events [3] and technical

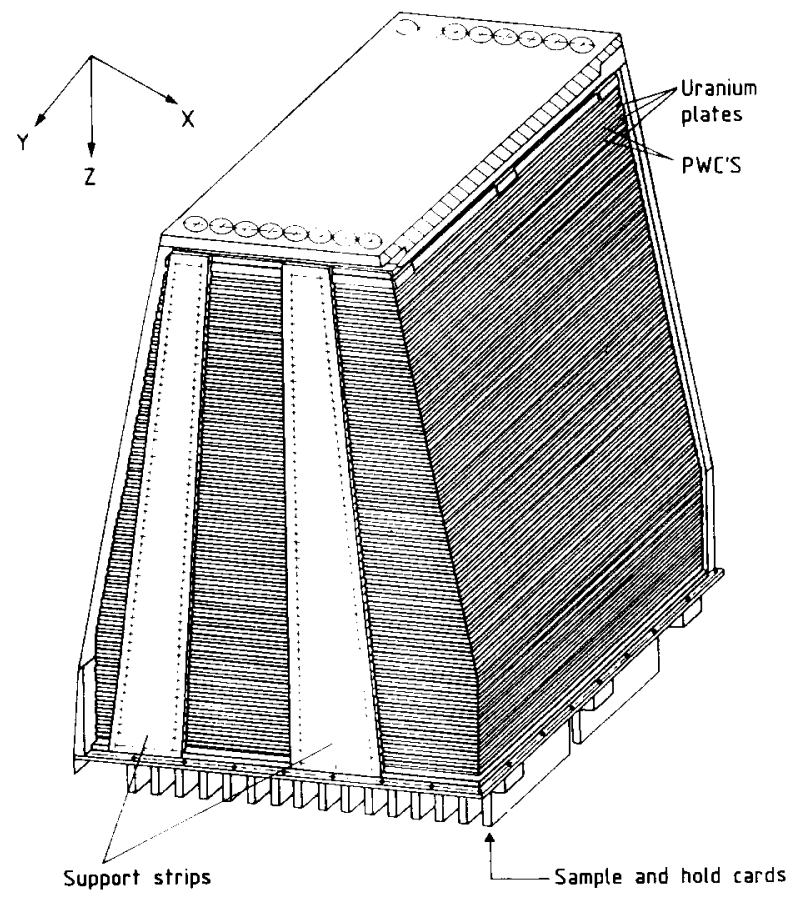

Fig. 1. The perspective view of the module with the outer cover removed. This shows the interleaved ${ }^{238} \mathrm{U}$ plates and wire chambers.

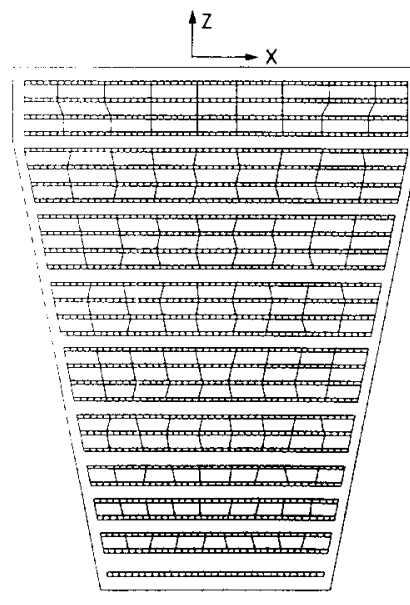

(a)

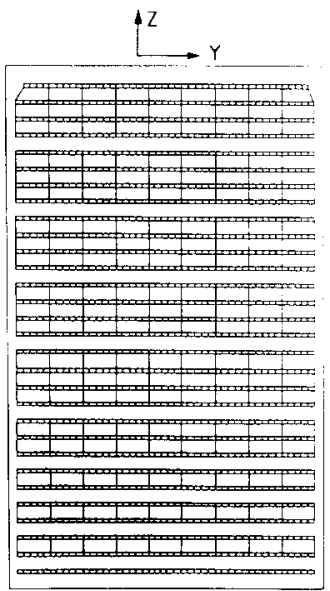

(b)
Fig. 2. The wire grouping scheme in the module: (a) grouping for $X$-towers; (b) grouping for $Y$-towers.

considerations, a scheme for grouping the wires inside the module was chosen. For accurate measurement of the energy flow of hadron events the 2763 wires in the module were grouped into 180 towers. There are 10 segments of towers along the beam or Z-direction (which is the radial direction in the L3 calorimeter), as shown in fig. 1. There are 9 segments in both the $X$ - and $Y$-directions. The towers with wires parallel to the $X$-direction are called $Y$-towers (since they measure $Y$ ), and the definition of $X$-towers is similar. Each tower contains between 3 and 27 wires ganged in parallel. Fig. 2 diagrams the scheme of the wire grouping in the module. On the assembled L3 experiment, cylindrical coordinates $R, \Phi$ and $z$ are appropriate. These coordinates are related to the above single module coordinates with $R \equiv Z, \Phi \equiv X$ and $z \equiv Y$.

\section{Signal digitization}

For the 1986 test beam data, the electrical signals generated by the proportional wire chambers were amplified and digitized using a modified version of the sample and hold system described in ref. [10]. This system consists of several components: a gated sample and hold card mounted directly to the hadron calorimeter module, an analog data bus, and a 12-bit ADC to measure the voltage on each sample and hold capacitor. A schematic of the circuit is shown in fig. 3.

The essential difference between the system in ref. [10] and the system which was used here is the addition of a fast amplifier and gate to the front end of the sample and hold cards. This allowed the application of a $400 \mathrm{~ns}$ gate in order to suppress spurious signals due to the uranium radioactivity. The signal was then de- 


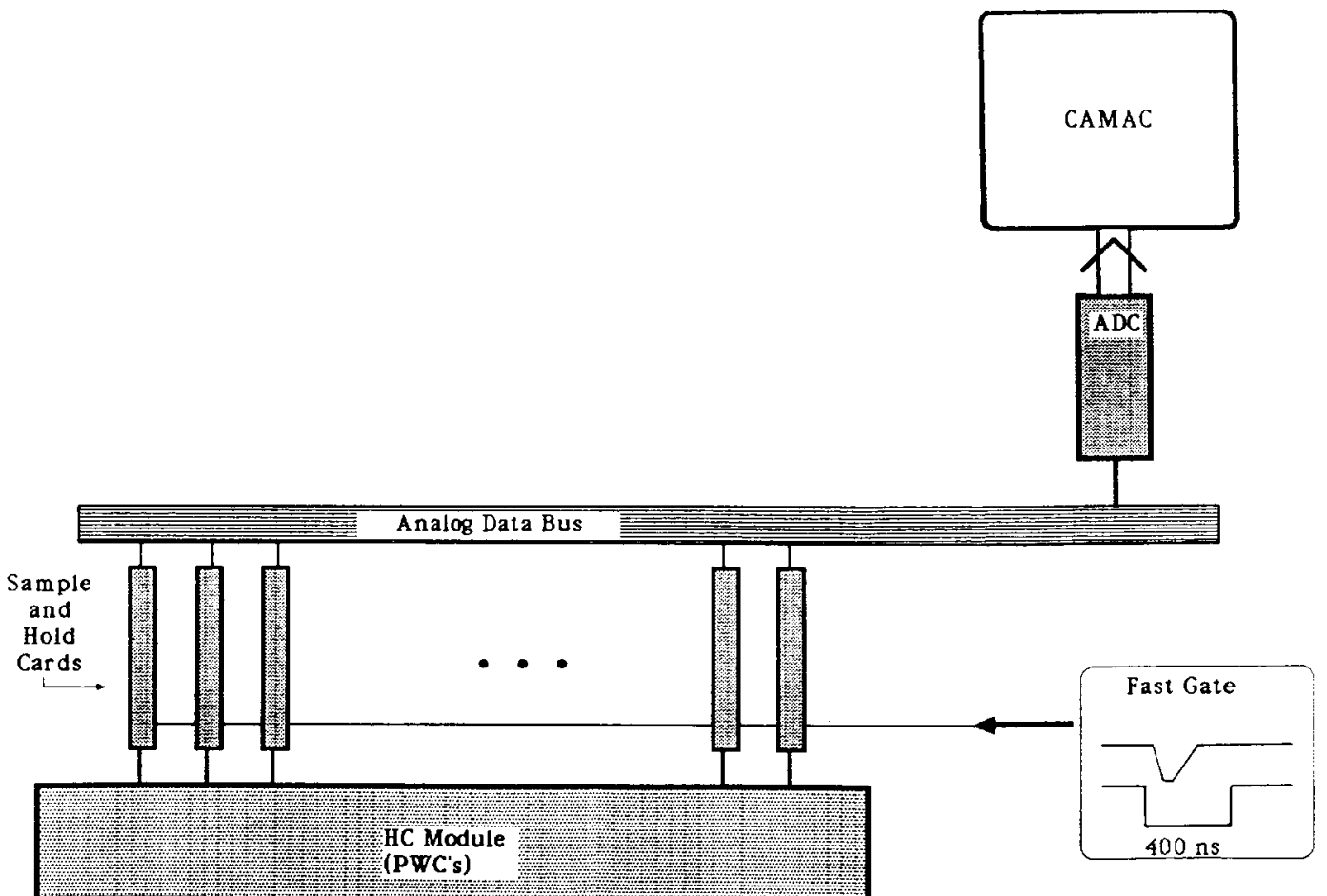

Fig. 3. The ADC system used to digitize the calorimeter signals. Twenty-four 8-channel gated sample and hold cards are used to sample the charge from the PWCs. The voltage on each sample and hold capacitor is then measured by a 12-bit ADC which is multiplexed to each 8-channel card.

layed by a 400 ns delay line before being applied to the hold storage capacitor for subsequent digitization.

The digitized signals were then read out by a VME CAMAC controller, passed through a monitoring VAX750 , and finally written onto magnetic tape for further analysis.

\section{Experiment setup}

The beam test was carried out in the $\mathrm{X} 3$ beamline of the CERN SPS in the autumn of 1986. This was a tertiary beam fed by $210 \mathrm{GeV}$ protons striking a beryllium target. It provided particles which range from 2 to $50 \mathrm{GeV}$ in energy. The momentum spread of the beam, $\Delta p / p$, was less than $1 \%$.

The particle trigger was designed to select either electrons, pions, or muons of a given momentum. Beam particles were identified by a pair of scintillation counters, S1 and S2, in the beamline. Electrons were selected by using a pair of threshold Cherenkov counters, $\mathrm{Cl}$ and $\mathrm{C} 2$. Penetrating muons were selected by using an 80 $\mathrm{cm}$ thick iron block as an absorber followed by a 0.25 $\mathrm{m}^{2}$ scintillation trigger counter $\mathrm{S} 3$. The particle trajecto-

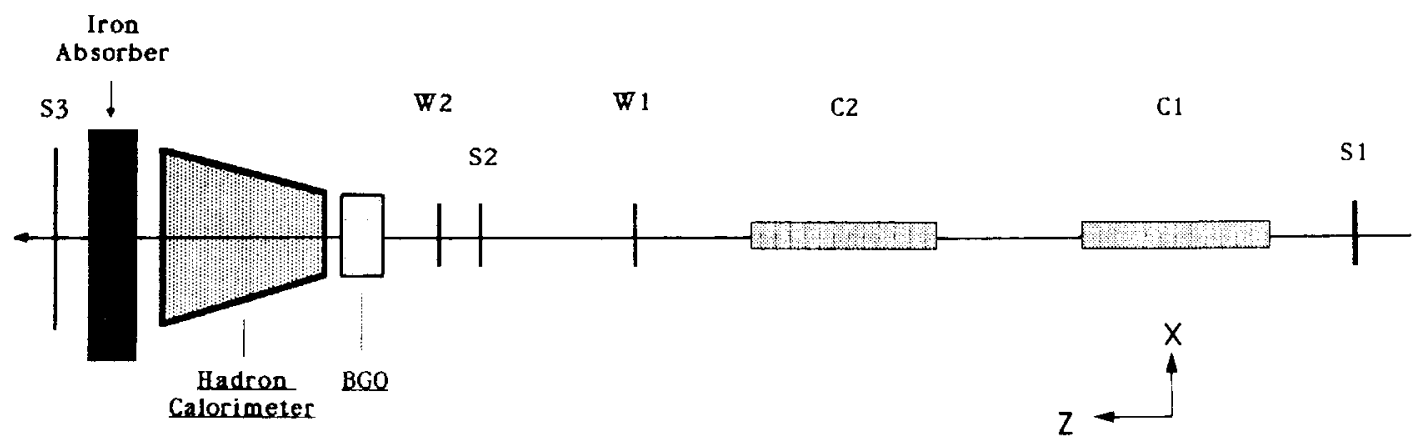

Fig. 4. The X-3 beamline consisted of two beam definition scintillation counters (S1 and S2), two wire chambers for measuring the particle trajectories (W1 and W2), two Cherenkov counters for detecting electrons (C1 and C2), and an iron absorber followed by a scintillator (S3), for detecting muons. 
ries were defined by a series of beamline multiwire proportional chambers, W1 and W2, with $1 \mathrm{~mm}$ wire spacing. A schematic of the beamline of experimental setup II is shown in fig. 4 . There was a $10 \times 10 \mathrm{BGO}$ crystal matrix in front of the module. The lenght of each BGO crystal was $24 \mathrm{~cm}$, which equals 22 radiation lengths and 1.1 proton absorption lengths. The front face of each crystal was $2 \mathrm{~cm} \times 2 \mathrm{~cm}$, and the back face $3 \mathrm{~cm} \times 3 \mathrm{~cm}$. The details of the structure of the BGO matrix and its performance have been described elsewhere [6,7]. The beamline passed through the center of the BGO matrix crystal and the center of the uranium module. Experimental setup I was the same as setup II with the BGO matrix removed.

\section{Calibration}

The pedestals of the tower readout channels were determined from random trigger runs (with high voltage on) by averaging the counts in channels which were above half of the peak value in the pulse height distributions. In the pulse height distributions, the tail corresponds to uranium radioactivity signals, shown in fig. 5 $(\mathrm{hv}=-1728 \mathrm{~V})$. The frequency of uranium noise was also calculated from the tail. The average rate of uranium signals per trigger per module is 2.25 . The mean value of uranium noise per module per trigger is $0.25 \mathrm{GeV}$ in equivalent hadron energy. During data analysis, the averaged equivalent noise energy was subtracted from measured energies deposited in the hadron calorimeter

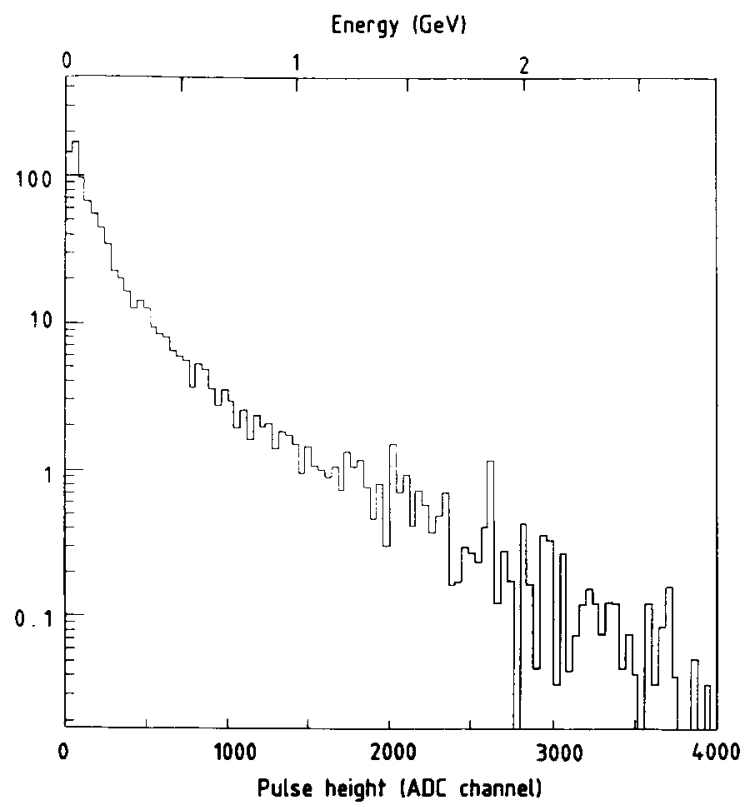

Fig. 5. The energy spectrum of uranium noise measured in random trigger runs for a single channel in the module.

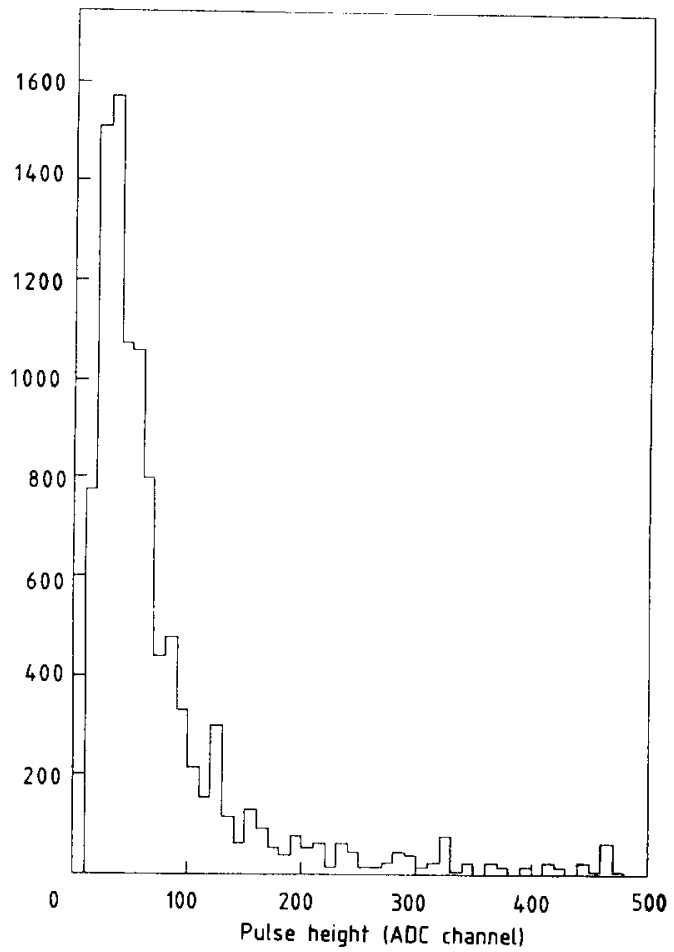

Fig. 6. The pulse height distributions of muons in a tower with one chamber plane.

module. This distribution was used as input to the Monte Carlo calculation to simulate the uranium noise.

During test beam runs, all towers were scanned with muon beams. By analyzing the muon data, the intercalibration constants between towers were obtained. To

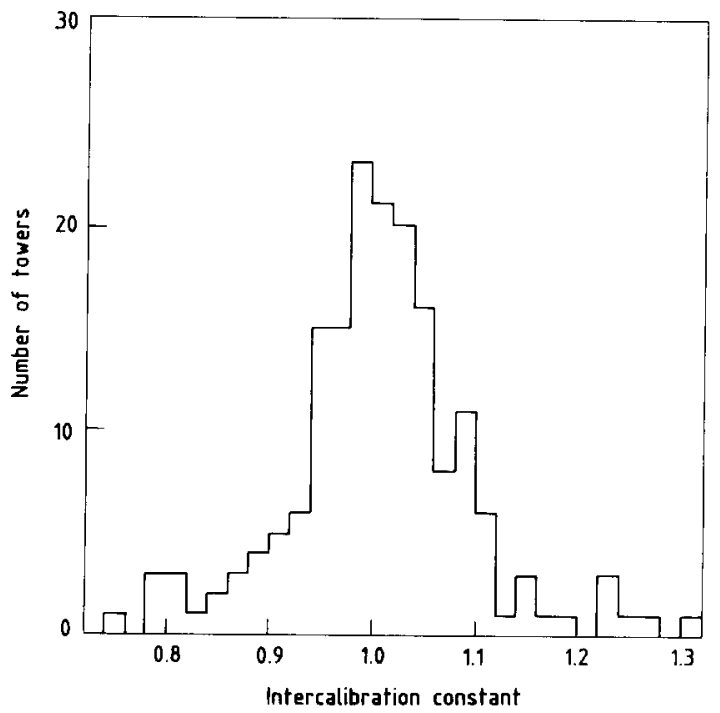

Fig. 7. The distribution of the intercalibration constants in the module from muon spectra. 
obtain clean signals of minimum ionizing particles, the high voltage in the muon runs was slightly higher than in the data taking runs. Fig. 6 is the pulse height distribution of minimum ionizing particles of momentum $20 \mathrm{GeV} / c$ for a tower with one chamber plane at $\mathbf{h v}=-1728 \mathrm{~V}$. The response to a "minimum ionizing particle" (mip) was defined to be the average value of the counts in channels which are above half of the peak value in the pulse height distributions of muons with a momentum of $20 \mathrm{GeV} / c$ divided by the number of chamber planes in the tower. A muon calibration for the central towers was also obtained in the course of the hadron data runs from non-interacting particles at the lower high voltage of $-1675 \mathrm{~V}$. The measurement shows that on average a mip in one proportional chamber corresponds to 29.1 ADC counts for $h v=-1675 \mathrm{~V}$, and to $41.6 \mathrm{ADC}$ counts for $\mathrm{hv}=-1728 \mathrm{~V}$. The intercalibration constant of a tower is defined to be the number of ADC channels per mip in the tower divided by the average mip value in the whole module. Fig. 7 is the distribution of the intercalibration constants of the towers for the beam test period where BGO matrix + one hadron calorimeter module were used. The data show that the pedestals and the intercalibration constants of the module are stable within $\pm 10 \%$ between calibration runs.

During the data analysis, all measured ADC channels were rescaled one by one according to the intercalibration constants, and converted into stardard values in units of mip.

The precise pedestal and gain of each BGO crystal were used to calculate the energy deposited in the BGO calorimeter in setup II. The calibration of the BGO crystals has been described in ref. [7].

\section{Monte Carlo simulation}

The two test beam setups were simulated by Monte Carlo computations based on the general simulation framework GEANT3 [8,9]. GEANT3 is a system of detector description and simulation tools. It describes an experimental setup in a rather efficient and simple way. To simulate the response of a detector, GEANT3 controls the transport of particles through various regions of the detector setup, taking into account the geometric volume boundaries and all physical effects due to the nature of the particles themselves, and to their interactions with matter. The program then records the elements of the particle trajectories and the response in the sensitive medium.

The geometries of the BGO $10 \times 10$ matrix, including walls and a few dead crystals, and the hadron calorimeter module were carefully described by GEANT3. The electromagnetic showers and hadronic cascades both in the BGO crystal matrix and the hadron calorimeter module were simulated in great detail. The kinetic energy cuts for the simulation of gammas, electrons, charged hadrons, neutral hadrons and muons were $1 \mathrm{MeV}$. The light propagation and the light collection efficiency in BGO crystals were simulated. The values of deposition energy in tubes within a tower were accumulated according to the scheme of wire grouping.

The measured beam width and the momentum spread of the beam were simulated in the Monte Carlo calculations.

Based on the results of the random trigger runs, the uranium noise in the hadron calorimeter was simulated in detail. The noise frequency of each tower was carefully calculated according to the total sensitive area of tubes in the tower. The deposition energy was generated according to the measured energy spectrum of the uranium noise in random trigger runs (fig. 5). The spectrum is independent of the size of a tower.

\section{Energy resolution and linearity}

The energy linearity and resolution were measured using setup I for pions of energies 6,10 and $20 \mathrm{GeV}$, and setup II for pions of energies of 4,10 and $20 \mathrm{GeV}$.

\section{I. Setup I (without BGO matrix)}

The measured energy response of setup I for pions of energies of 6,10 and $20 \mathrm{GeV}$ is shown in fig. 8. Each energy point contains about $6 \mathrm{~K}$ events. The measurements show that on average 36.0 mips correspond to 1 $\mathrm{GeV}$ hadron energy deposition, or $27.8 \mathrm{MeV} / \mathrm{mip}$. The average energy response is shown in fig. 9 . The error bars contain both the statistical and systematic error.

When the response to hadrons is compared to the response to muons cited above (section 5) a weighting factor can be deduced. Thus, from the definition of a mip and the value for the most probable energy loss of a minimum ionizing particle [11] passing through $5 \mathrm{~mm}$ of $\mathrm{U}$ and $2 \mathrm{~mm}$ of brass (taken as $12 \mathrm{MeV}$ ), it is seen that $G_{\mathrm{H}}=(27.8 \mathrm{MeV} / \mathrm{mip}) /(12 \mathrm{MeV} / \mathrm{mip})$, or $G_{\mathrm{H}}=$ 2.31.

The energy resolution was defined in terms of the full width at half maximum (FWHM). For purposes of comparison with equivalent Gaussian parameters, the value of FWHM/2.36 is used here. Generally a Gaussian fit gives a value of the standard deviation greater than the value taken from FWHM/2.36. The resolution for the three energies was $30.5 \pm 3 \%$ at $6 \mathrm{GeV}, 21 \pm 2 \%$ at $10 \mathrm{GeV}$ and $18 \pm 2 \%$ at $20 \mathrm{GeV}$.

Fig. 10 shows the energy resolution of setup I as a function of pion energy, compared with the predictions of the Monte Carlo simulation. The triangles are the data, and the points are the predictions from Monte Carlo calculation. The error bars of the Monte Carlo 

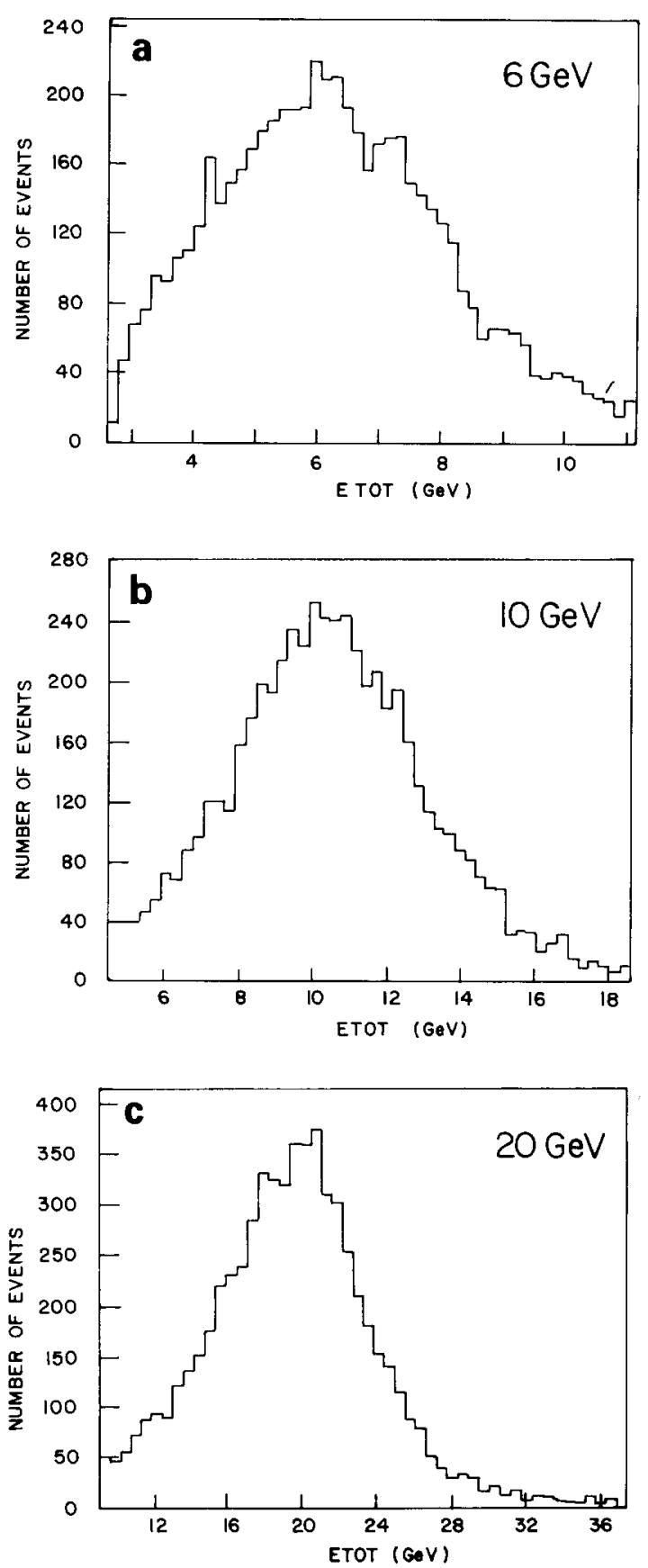

Fig. 8. The energy response of pions for setup I (without BGO matrix): (a) $6 \mathrm{GeV}$, (b) $10 \mathrm{GeV}$, (c) $20 \mathrm{GeV}$.

prediction come mainly from the systematic errors in the simulation, since the uranium fission and the gas amplification in the hadron calorimeter were not well understood. The fit to the measured energy resolution as a function of the beam energy gave the relation:

$\sigma_{E} / E=(61 / \sqrt{E}+3) \%$,

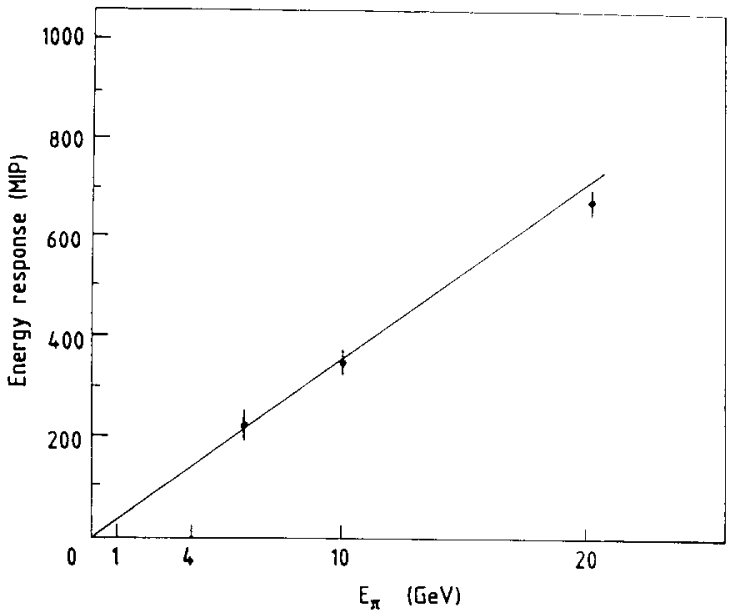

Fig. 9. The linearity of energy response of pions for setup I. The line is fit to the 6 and $10 \mathrm{GeV}$ points and constrained to pass through the origin.

where $E$ on the right hand side is expressed in $\mathrm{GeV}$.

\subsection{Setup II (with BGO matrix)}

In setup II, a pion deposits its energy in both the BGO matrix and the hadron calorimeter. The total energy of an event, $E_{\mathrm{TOT}}$, can be expressed as:

$E_{\text {TOT }}=G_{\mathrm{B}} E_{\mathrm{b}}+G_{\mathrm{H}} E_{\mathrm{h}}$,

where $E_{\mathrm{b}}$ is the energy deposited in the BGO matrix as calibrated by incident electron beams, and $E_{\mathrm{h}}$ is the energy deposited in the hadron calorimeter as calibrated by muons and non-interacting pions. $G_{\mathrm{B}}$ and $G_{\mathrm{H}}$, called the geometry factors for the $\mathrm{BGO}$ and the hadron calorimeter respectively, are two free parameters to be fit. To fit the geometry factors, we took the pion data sample from the beam test, and calculated $E_{\mathrm{b}}$ and $E_{\mathrm{h}}$. The best values of $G_{\mathrm{B}}$ and $G_{\mathrm{H}}$ are obtained by minimizing the following function, which will be called $\chi^{2}$ here:

$\chi^{2}\left(G_{\mathrm{B}}, G_{\mathrm{H}}\right)=\sum \frac{\left(E_{\text {beam }}-E_{\mathrm{TOT}}\right)^{2}}{\epsilon^{2}}$,

where $\epsilon^{2}=\sqrt{E_{\text {beam }} E_{\text {TOT }}}$, and the summation is over all events in the sample. In eq. (3) and in the definition of $\epsilon$ the energies $E_{\text {beam }}$ and $E_{\text {TOт }}$ are taken as pure numbers, in units of $1 \mathrm{GeV}$. It is observed that this definition of $\chi^{2}$ does not bias the procedure when compared with the conventional definition. For an individual event, $\epsilon$ is dominated by the fluctuations of the hadron shower. The optimized total energy distribution is the $E_{\text {TOT }}$ calculated by the values of $G_{\mathrm{B}}$ and $G_{\mathrm{H}}$ according to eq. (2). The energy resolution is defined to be the FWHM of the total energy distribution divided by 2.36 . Fig. 11 shows the energy resolution of setup II as a 


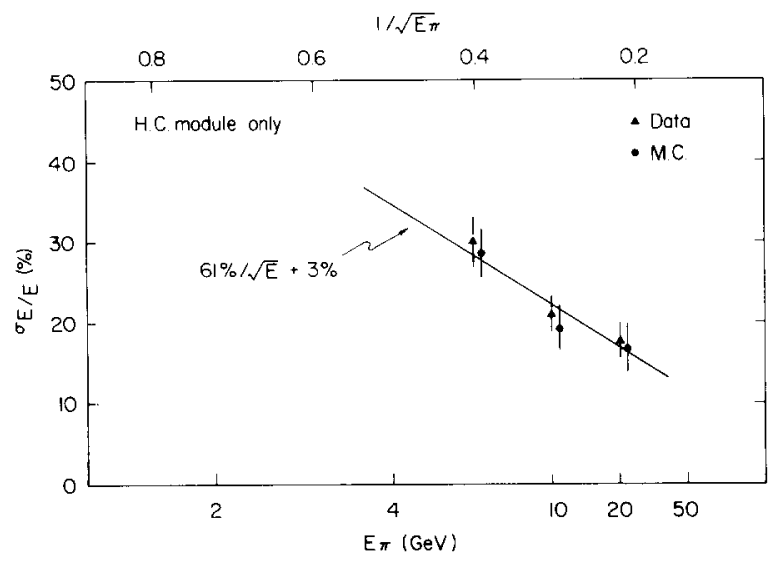

Fig. 10. The energy resolution of pions for setup I (without BGO matrix).

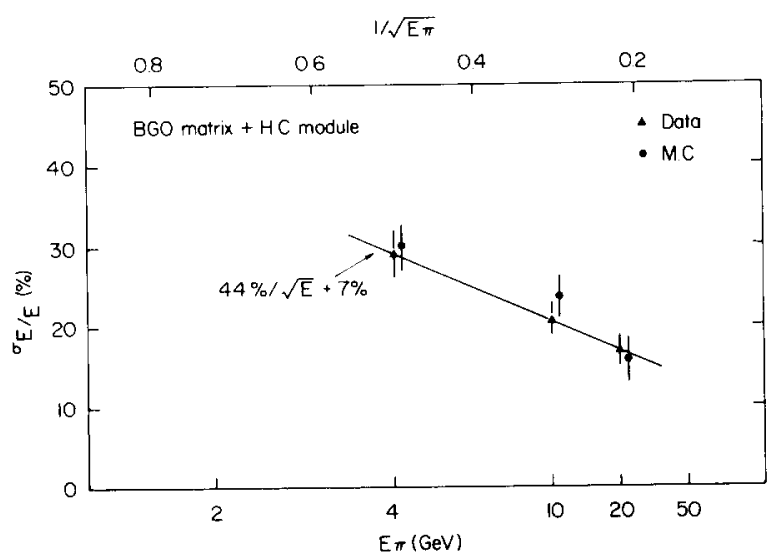

Fig. 11. The energy resolution of pions for setup II (with BGO matrix).

function of pion energy. There are about $45 \times 10^{3}$ events at each energy point. The error bars of the Monte Carlo prediction contain $3 \%$ uncertainty from the simulation. There are $1 \%$ uncertainties in fitting the geometry factors in both the data and Monte Carlo prediction. The fit to the measured energy resolution as a function of the beam energy gave the relation:

$\sigma_{E} / E=(44 / \sqrt{E}+7) \%$,

where $E$ on the right hand side is expressed in $\mathrm{GeV}$. The geometry factors depend only slightly on the beam

Table 1

Energy resolution and geometry factors of setup II

\begin{tabular}{clll}
\hline$E_{\text {beam }}[\mathrm{GeV}]$ & $G_{\mathrm{B}}$ & $G_{\mathrm{H}}$ & $\sigma_{E} / E[\%]$ \\
\hline 4 & $2.05 \pm 0.21$ & $2.45 \pm 0.25$ & $29.0 \pm 3.0$ \\
10 & $2.02 \pm 0.20$ & $2.36 \pm 0.24$ & $21.0 \pm 2.0$ \\
20 & $1.90 \pm 0.19$ & $2.38 \pm 0.24$ & $17.0 \pm 2.0$ \\
\hline
\end{tabular}
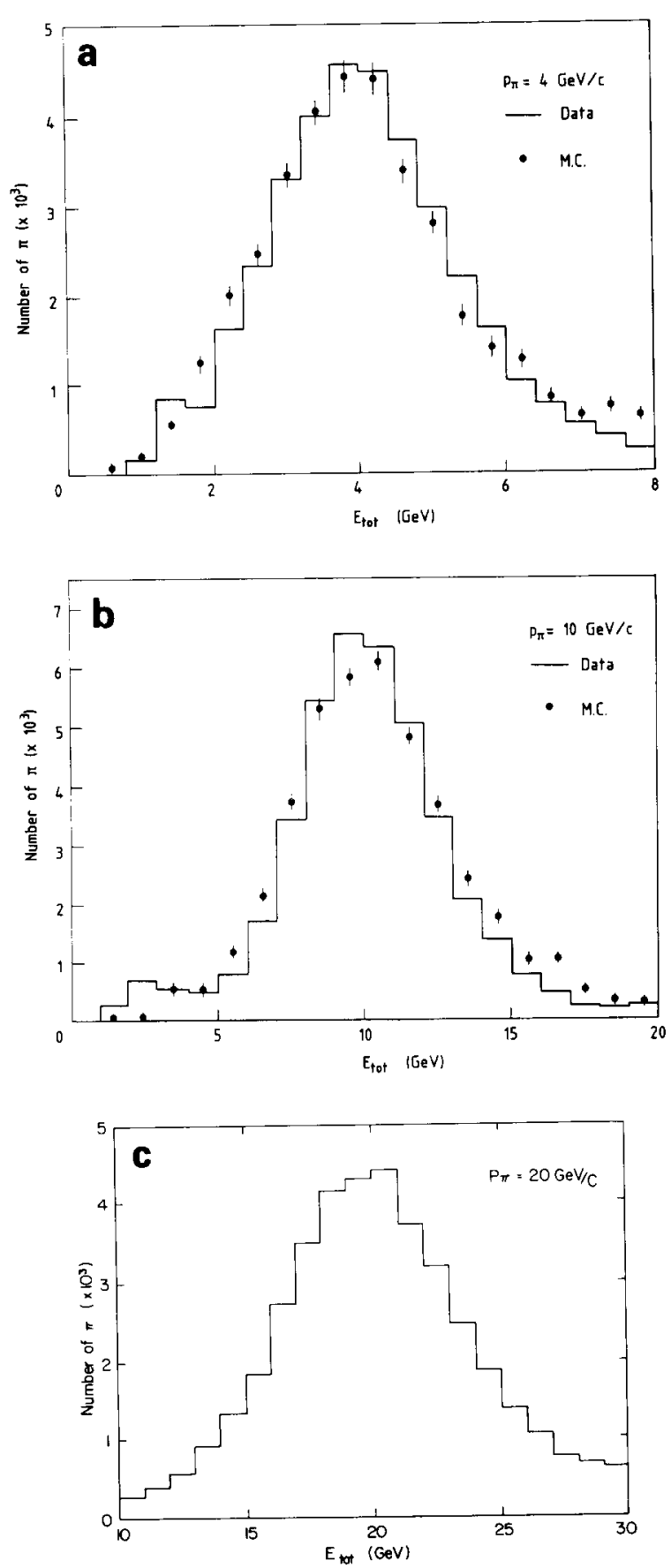

Fig. 12. The total energy distributions of pions and comparison with the Monte Carlo simulation for setup II. The error bars contain the statistical errors only. (a) $4 \mathrm{GeV}$, (b) $10 \mathrm{GeV}$, (c) 20 $\mathrm{GeV}$.

energy, which means that setup II has a good linearity to measure the energy of pions. The values of $G_{\mathrm{B}}$ and $G_{\mathrm{H}}$ as well as the energy resolutions for the three 
energies are tabulated in table 1 . Variation of $G_{\mathrm{B}}$ and $G_{\mathrm{h}}$ by $\pm 10 \%$ from their optimum values increased $\sigma_{E} / E$ by less than $1.5 \% . G_{\mathrm{B}}$ is greater than 1 due to side energy leakage of the BGO matrix, light saturation of heavy charged particles in the BGO crystals and nuclear binding energy losses in hadron showers. We should point out that $G_{\mathrm{B}}$ is not simply the ratio of the $\mathrm{e} / \pi$ response.

As the resolution depends only slowly on $G_{\mathrm{B}}$ and $G_{\mathrm{H}}$, it would be more practical to fix $G_{\mathrm{B}}$ and $G_{\mathrm{H}}$ independent of beam energy. When $G_{\mathrm{B}}$ is fixed at 2.09 (the average of $G_{\mathrm{B}}$ at the three energies) and $G_{\mathrm{H}}$ is fixed at 2.31 (from the calorimeter calibration without BGO), the energy resolution is $29.3 \%$ with a mean energy of $3.93 \mathrm{GeV}$ at $E_{\text {beam }}=4 \mathrm{GeV}, 21.2 \%$ with a mean energy of $10.0 \mathrm{GeV}$ at $E_{\text {beam }}=10 \mathrm{GeV}$ and $17.5 \%$ with a mean energy of $20.4 \mathrm{GeV}$ at $E_{\text {beam }}=20 \mathrm{GeV}$.

Figs. $12 a-c$ show the measured total energy distribution of pions for beam energies of 4,10 and $20 \mathrm{GeV}$. Figs. 12a and $\mathrm{b}$ also show the comparison with the predictions of the Monte Carlo simulation. The histograms are the data, and the points are the predictions from the Monte Carlo calculation. The error bars contain only the statistical error. The data and the prediction of the Monte Carlo calculation are in agreement within errors. Fig. 13 shows the energy deposited in the BGO matrix vs the energy deposited in the hadron calorimeter module for $20 \mathrm{GeV}$ pions, where $E_{\mathrm{BGO}}=$ $G_{\mathrm{B}} E_{\mathrm{b}}$, and $E_{\mathrm{H} . \mathrm{C} .}=G_{\mathrm{H}} E_{\mathrm{h}}$. Table 2 summarizes $E_{\mathrm{BGO}} / E_{\mathrm{TOT}}$ and $E_{\mathrm{H.C}} / E_{\mathrm{TOT}}$, the fractional energy de-

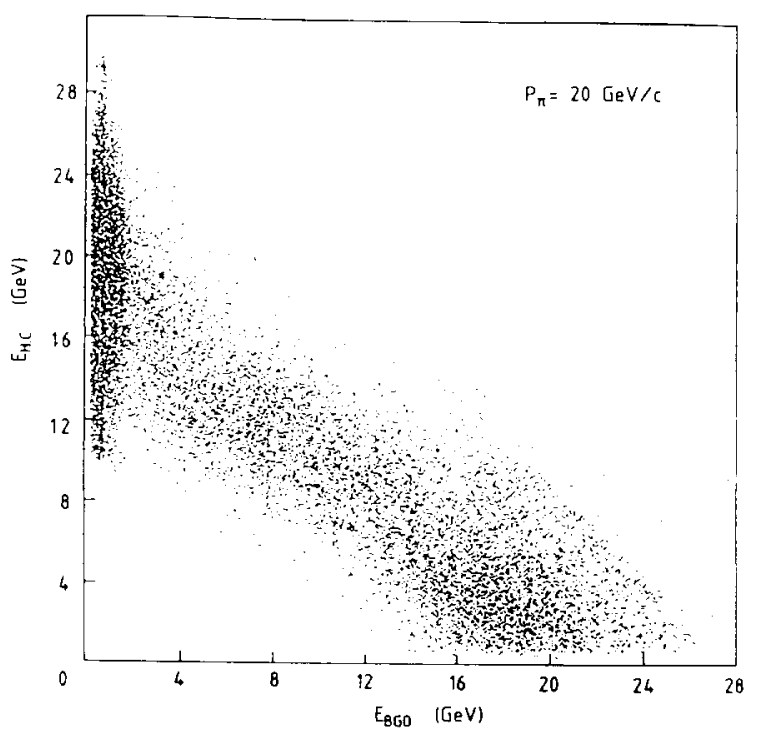

Fig. 13. The energy deposited in the $\mathrm{BGO}$ matrix, $E_{\mathrm{BGO}}=$ $G_{\mathrm{B}} E_{\mathrm{b}}$, vs the energy deposited in the hadron calorimeter module, $E_{\mathrm{H} . \mathrm{C}}=G_{\mathrm{H}} E_{\mathrm{h}}$, for pions of $20 \mathrm{GeV}$.
Table 2

Fractional observed energy depositions in the BGO and in the hadron calorimeter module

\begin{tabular}{llll}
\hline$P_{\text {beam }}[\mathrm{GeV} / c]$ & $E_{\mathrm{BGO}} / E_{\mathrm{TOT}}[\%]$ & $E_{\mathrm{H} . \mathrm{C}} / E_{\mathrm{TOT}}[\%]$ \\
\hline 4 & Data & $52.3 \pm 0.3$ & $47.7 \pm 0.3$ \\
& $\mathrm{MC}$ & $52.8 \pm 0.7$ & $47.2 \pm 0.7$ \\
10 & Data & $46.4 \pm 0.3$ & $53.6 \pm 0.3$ \\
& MC & $48.5 \pm 0.7$ & $51.5 \pm 0.7$ \\
20 & Data & $34.8 \pm 0.4$ & $65.2 \pm 0.4$ \\
& MC & $33.0 \pm 0.9$ & $67.0 \pm 0.9$ \\
\hline
\end{tabular}

posited in $\mathrm{BGO}$ and the hadron calorimeter as a function of beam energy in both the measured data and the Monte Carlo calculation. The Monte Carlo predictions are in agreement with the data.

The energy resolutions of the two setups are similar. This shows that the energy resolution of charged pions is similar both with and without the BGO matrix in front of the hadron calorimeter.

\section{Position resolution}

The positions of the impact points of pions were reconstructed from the energy deposition in both BGO crystals and hadron calorimeter towers by the center of gravity method for setup II. For the reconstruction of the position in the hadron calorimeter, we require a minimum energy deposited in the module of $1 \mathrm{GeV}$. The tower position was defined to be the average position of the wires in the tower. The wire directions in two neighboring planes are perpendicular, which permits an adequate position determination for hadron vertices.

By comparing the impact points of pions reconstructed from the measurements in the beamline proportional chambers, the position resolutions of both the BGO matrix and the uranium module were calculated. The measured position resolutions are shown in table 3 compared with the predictions of Monte Carlo calcula-

Table 3

Pion position resolution

\begin{tabular}{lllr}
\hline$P_{\text {beam }}[\mathrm{GeV} / c]$ & $\sigma_{\mathrm{BGO}}[\mathrm{mm}]$ & \multicolumn{1}{c}{$\sigma_{\mathrm{H.C}}[\mathrm{mm}]$} \\
\hline 4 & Data & $6.4 \pm 0.3$ & $10.1 \pm 0.5$ \\
& MC & $6.1 \pm 0.7$ & $7.5 \pm 0.7$ \\
10 & Data & $5.6 \pm 0.3$ & $10.6 \pm 0.5$ \\
& MC & $5.4 \pm 0.5$ & $10.1 \pm 1.0$ \\
20 & Data & $4.7 \pm 0.2$ & $8.4 \pm 0.4$ \\
& MC & $5.7 \pm 0.5$ & $10.7 \pm 1.0$ \\
\hline
\end{tabular}


tions. The error bars of the Monte Carlo predictions come from the systematic error of the shower simulation.

\section{Conclusions}

A prototype of the L3 hadron calorimeter module with uranium sampling and gas proportional chamber readout was built and tested. The test beam results of the module with a pion beam show satisfactory performance in energy resolution, energy linearity and position resolution. The energy resolution of the hadron calorimeter module with and without the BGO matrix in front are similar. The calculations from the GEANT Monte Carlo program are in agreement with the test beam data.

\section{Acknowledgements}

The beam test of setup II was carried out with a $10 \times 10 \mathrm{BGO}$ matrix in front of the hadron calorimeter module. We would like to thank all members of the L3 BGO group for their collaboration, including the data taking and providing the programs to analyze the BGO data.

We would like to thank Prof. S.C.C. Ting for his support. We would also like to thank Drs. A. Arefiev,
Y. Galaktionov, Yu. Kamyshkov, A. Kunin, V. Shoutko, M. Capell, M. Chen, C. Dionisi, F. Carminati, B. Clare and $\mathrm{H}$. Newman for useful discussions. We acknowledge Prof. M. Longo for electronics design. The authors from the University of Michigan would like to thank US NSF for their support under grant PHY-8409615.

\section{References}

[1] L3 collaboration, Technical Proposal of L3, CERN/ LEPC/83-5 (May, 1983) unpublished.

[2] L3 collaboration, The Construction of L3 (April, 1985) unpublished.

[3] He Sheng Chen, Nucl. Instr. and Meth. A256 (1987) 261.

[4] F. Celletti et al., Nucl. Instr. and Meth. A225 (1984) 493.

[5] A. Arefiev et al., Nucl. Instr. and Meth. A245 (1986) 71; Yu. Galaktionov et al., Nucl. Instr. and Meth. A251 (1986) 258

[6] J.A. Bakken et al., Nucl. Instr. and Meth. A228 (1985) 294.

[7] J.A. Bakken et al., Nucl. Instr. and Meth. A254 (1987) 535.

[8] R. Brun, F. Bruyant, M. Maire, A. McPherson and P. Zanarini, GEANT3, CERN DD/EE/84-1, unpublished.

[9] H. Fesefeldt, PITHA 85-02, unpublished.

[10] R.C. Ball et al., Nucl. Instr. and Meth. A197 (1982) 371.

[11] The absorption length is defined as the nuclear interaction length $\lambda_{I}$, see: Particle Data Properties, Phys. Lett. B170 (1986) 38 . 